\title{
Substrate-Selective Morphology of Cesium lodide Clusters on Graphene
}

\author{
Nilesh Vats,* Yi Wang, Suman Sen, Sven Szilagyi, Hannah Ochner, Sabine Abb, Marko Burghard, \\ Wilfried Sigle, Klaus Kern, Peter A. van Aken, and Stephan Rauschenbach
}

Cite This: ACS Nano 2020, 14, 4626-4635

Read Online

ACCESS |

|lll Metrics \& More

Article Recommendations

Supporting Information

ABSTRACT: Formation and characterization of low-dimensional nanostructures is crucial for controlling the properties of twodimensional (2D) materials such as graphene. Here, we study the structure of low-dimensional adsorbates of cesium iodide (CsI) on freestanding graphene using aberration-corrected transmission electron microscopy at atomic resolution. CsI is deposited onto graphene as charged clusters by electrospray ion-beam deposition. The interaction with the electron beam forms two-dimensional CsI crystals only on bilayer graphene, while CsI clusters consisting of $4,6,7$, and 8 ions are exclusively observed on single-layer graphene. Chemical characterization by electron energy-loss spectroscopy imaging and precise

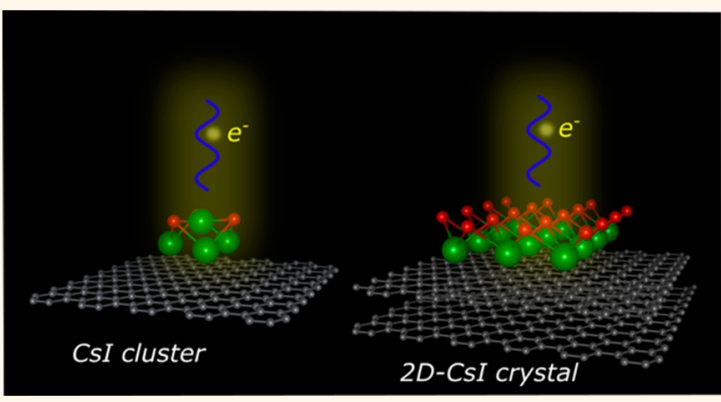
structural measurements evidence the possible influence of charge transfer on the structure formation of the CsI clusters and layers, leading to different distances of the Cs and I to the graphene.

KEYWORDS: graphene, electrospray ion-beam deposition, transmission electron microscopy, mass spectrometry, alkali halide atomic clusters and two-dimensional crystals

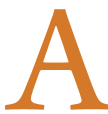

dsorption of atomic and molecular species on surfaces is a reliable and widely rehearsed approach toward tuning the properties of low-dimensional materials such as graphene. The formation of assemblies of atomically defined adsorbates requires chemically pure coating in a well-controlled environment as well as the capability of atomic-scale characterization. Recent developments in TEM/STEM instrumentation $^{1-3}$ coupled with high-speed detectors has enabled observation of structure, dynamics, and chemistry of atoms/ molecules on surfaces at sub-angstrom resolution. ${ }^{4,5}$

Electron-beam-driven reactions allow the formation of chemical species with mechanisms fundamentally different from other external energy such as heating, light, or ion bombardment. ${ }^{6}$ During electron microscopy characterization on graphene, the electron beam can be used for induced manipulation of adsorbates to gain insights into substrateadsorbate interaction in real time and at the atomic level. ${ }^{7-9}$ Recent studies were able to show the creation of well-defined defects in graphene, ${ }^{10}$ rearrangement and graphitization of organic molecules, and etching of graphene under the influence of adsorbate atoms. ${ }^{11,12}$ Moreover, successful formation of ordered two-dimensional (2D) layers of metals via rearrangement under the electron beam using a $2 \mathrm{D}$ material surface as template has also been reported. ${ }^{13-15}$

While previous studies were able to demonstrate the formation of stable $2 \mathrm{D}$ crystals on atomically thin substrates, thus far, the influence of the substrate on morphology of alkali halide clusters and its 2D-crystal formation has not been reported. With the electron irradiation providing the energy source for the rearrangement of the adsorbates, ${ }^{9}$ the structure and morphology of the product will depend on the interaction with the substrate, which is providing the potential energy landscape that defines the reaction dynamics and hence the product. For graphene, this can be readily adjusted by changing the number of layers. ${ }^{16}$

In this work, we report how the morphology of cesium iodide clusters critically depends on the number of graphene layers when exposed to an electron beam in aberration-corrected transmission electron microscopy (AC-TEM, $80 \mathrm{kV}$ ). Initially, large nanoparticles of cesium iodide (CsI) are formed on freestanding graphene by electrospray ion-beam deposition (ESIBD). ${ }^{17,18}$ The sample is then exposed to the electron beam during high-resolution TEM (HRTEM) characterization. This results in formation of ordered 2D CsI crystals explicitly on

Received: January 8, 2020

Accepted: April 13, 2020

Published: April 13, 2020 
bilayer graphene (BLG) and atomic clusters of specific, molecular shape, on single-layer graphene (SLG).

We show that the combination of in-vacuum ES-IBD and electron-beam irradiation in TEM allows for a reliable formation of the low-dimensional nanostructures, by ensuring the purity of the coating through mass-to-charge ratio $(\mathrm{m} / z)$ filtering and precise control of the coverage. We are able to thoroughly characterize the formed 2D crystals structurally and chemically by electron energy-loss spectroscopy (EELS) mapping in probecorrected scanning transmission electron microscope (STEM) and observe the dynamic behavior of grain boundaries and multilayered crystallites. The chemical composition of the $2 \mathrm{D}$ crystals on bilayer graphene further allows us to infer the structure and composition of the atomic clusters.

\section{RESULTS AND DISCUSSION}

Sample Formation from 3D CsI Nanocrystals. Electrospray ionization of a CsI solution generates $(\mathrm{CsI})_{n} \mathrm{Cs}^{+}$ionic clusters $(n=1, \ldots, 6)$, which are analyzed by mass spectrometry (see Figure S1) and then deposited on freestanding graphene substrates via ES-IBD at a landing energy of $200 \mathrm{eV}$ per charge. The deposited charge of $454 \mathrm{pAh}$ (pico-amp-hour, $1 \mathrm{pAh}=6.25$ $\times 10^{6} \mathrm{e}^{-} / \mathrm{s}$ ) corresponds to approximately one monatomic layer of CsI on the surface of a TEM grid. After deposition, the samples were transferred to the TEM. A homogeneous distribution of bulk 3D cesium iodide nanocrystals is found in low-magnification TEM bright-field imaging (Figure 1b). The dark contrast of some particles is attributed to Bragg reflection indicative of crystalline order.

The formation of the $3 \mathrm{D}$ crystals can be attributed to the impact of the high kinetic energy CsI cluster ions during the deposition. While the chemical structure of the graphene is not affected, upon impact, the clusters likely disintegrate ${ }^{19}$ and the Cs and I adsorbates rearrange due to a transient mobility from

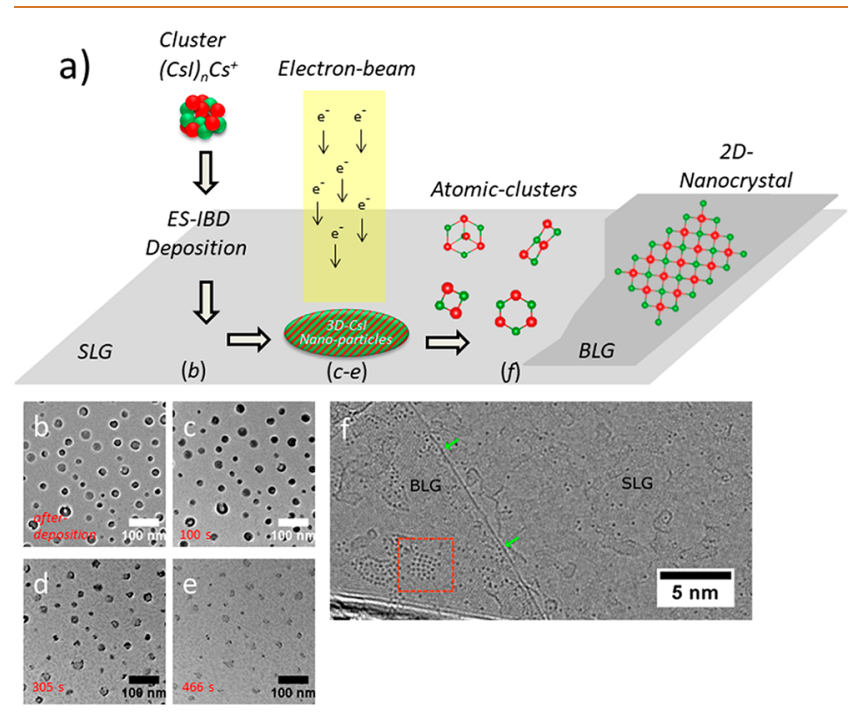

Figure 1. Sample formation. (a) Schematic representation of the main steps of the sample formation process including ES-IBD of $(\mathrm{CsI})_{n} \mathrm{Cs}^{+}$clusters, electron beam irradiation, and $2 \mathrm{D}$ crystals forming. (b-e) Time series of the decomposition of 3D CsI nanoparticles under electron beam irradiation at $80 \mathrm{kV}$ : (b) as prepared, (c) after $100 \mathrm{~s}$, (d) after $305 \mathrm{~s}$, (e) after $466 \mathrm{~s}$. (f) HRTEM image of Cs and I atoms in an SLG and BLG region of the sample separated by a step edge (green arrows). On BLG ordered CsI aggregates (red square). On SLG, Cs and I are present as adatoms. the impact energy. ${ }^{6,20}$ They agglomerate to form threedimensional (3D) nanocrystals with an average diameter of about $22 \mathrm{~nm}$ (Figure S1d). The chemical composition is confirmed by EELS acquired from individual nanoparticles (Figure S1c) in which adsorption edges of only cesium and iodine atoms are observed.

Alkali halides are among the most beam-sensitive inorganic materials. $^{21}$ During imaging, the $3 \mathrm{D}$ nanocrystals rapidly decompose (Figure 1c-e) during a time of approximately 8 min (refer to Video S1.1) at a total electron dose of $1.16 \times 10^{6} \mathrm{e} /$ $\mathrm{nm}^{2}$. The crystal structure of one such decomposing 3D CsI nanocrystal is shown in SI Figure S1.1. The fast Fourier transform (FFT) (Figure S1.1b) exhibits a $\{100\}$-CsI reflection at $0.45 \mathrm{~nm}$ and a $\{110\}-\mathrm{CsI}$ reflection at $0.32 \mathrm{~nm}$, viewed down its [001] axis, suggesting that the lattice constant of the observed 3D CsI nanocrystal is consistent with that of the bulk CsI, which corresponds to a $(\mathrm{CsCl}-b c c)$-type configuration. Moreover, the time-series HRTEM images of a decomposing 3D CsI nanoparticle are shown in (Figure $\mathrm{S} 1.2 \mathrm{a}-\mathrm{c}$ ), indicative of the crystal being reorganized while shrinking under the electron beam. In Figure S1.2d-g their line profiles along the (110) and (200) planes are plotted with their corresponding interplanar spacing $(d)$ values.

The decomposition occurs mainly due to radiolysis, ${ }^{22}$ leading to degradation by breaking of bonds and subsequent loss of mass and crystallinity (refer to SI Video S1.2). As a consequence, atomic species of Cs and I are emitted from decomposing 3D nanocrystals, some acting as sources of individual adsorbed Cs and I adatoms. Activated thermally or by the electron beam, Cs and I adatoms migrate on both single and bilayer graphene. By this, Cs and I adatoms are distributed evenly as clearly imaged by TEM (Figure 1f). In contrast to the 3D crystals, the adsorbates directly bonded to the graphene are stable under the electron beam. While most likely heated by the electron bombardment, which causes lateral mobility, the strongly coupled graphene acts as an effective sink for heat and charges generated by electron impact. $^{23,24}$

This creates a situation not unlike the one used for crystal growth from the gas phase. Cs and I atoms will encounter to form nucleation centers, which grow by aggregation of additional atoms. The overall growth process is complex, its outcome depending on many conditions such as the density of adatoms, the surface energies of graphene (SLG and BLG) and CsI, and their interface energy.

Figure 1f shows a boundary of single and bilayer graphene in an HRTEM image after prolonged exposure to the electron beam (see also Figure S2). The type of graphene can be identified based on the characteristic moire pattern visible only for BLG. Here, the influence of the interaction strength of the substrate becomes visible directly. We observe only a low density of individual adatoms on SLG, whereas on BLG a much higher density of adatoms is present, forming extended, twodimensional crystals (red square). In addition to clean areas of SLG and BLG, part of the surface is covered with graphitized organic contamination, likely remains of poly(methyl acrylate) (PMMA) used in the graphene transfer. On both SLG and BLG, adatoms adhere to the edges of holes or to other defects present on graphene such as vacancy defects or amorphous impurities.

2D Crystals of CsI on BLG. Two-dimensional CsI crystals were observed exclusively on BLG but never found on SLG. During HRTEM examination the 2D CsI crystals are formed upon prolonged electron exposure of a large area at an energy of $60 \mathrm{keV}$ using a parallel electron beam (see Figure S2). Residual 

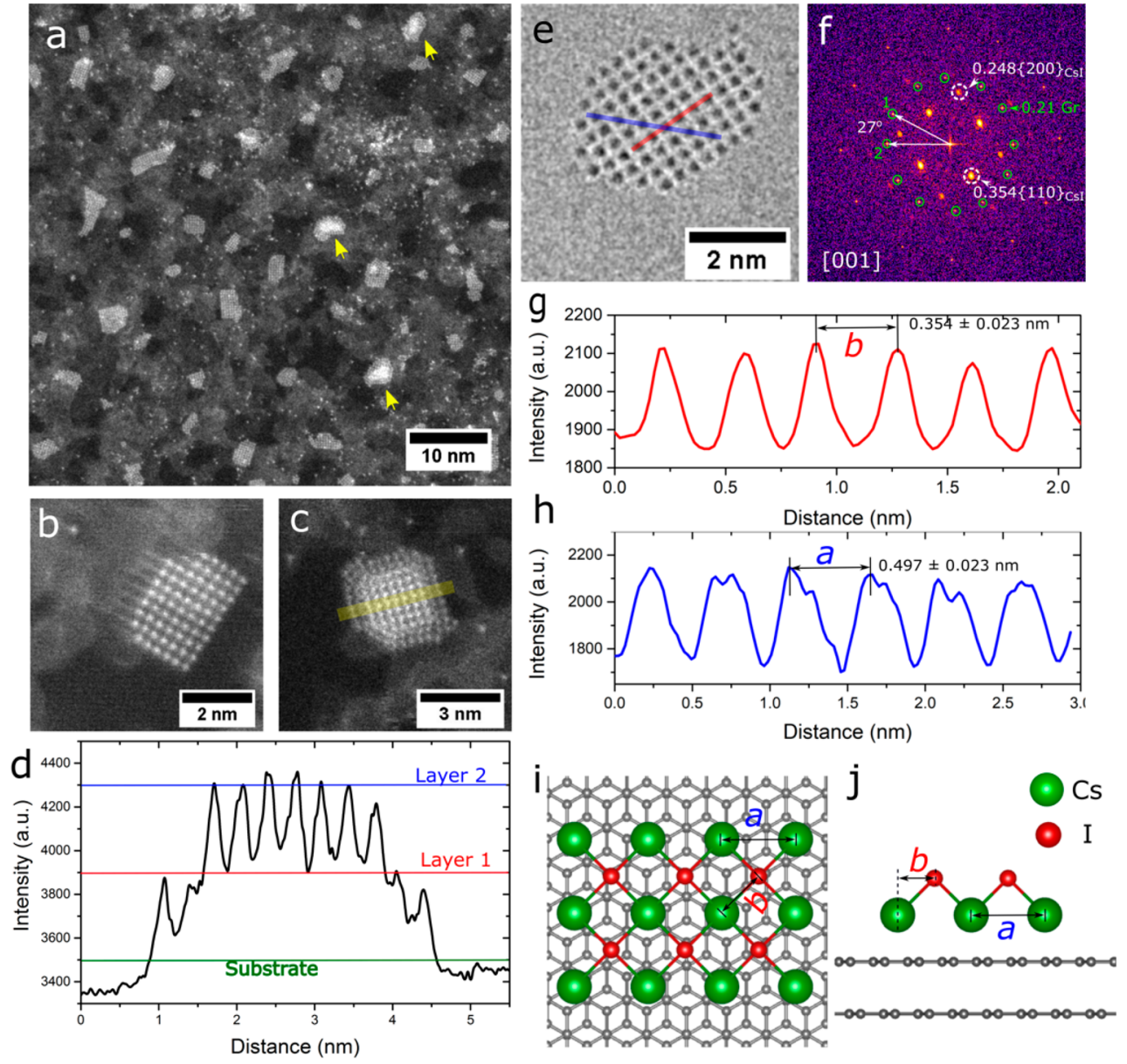

Figure 2. 2D CsI crystal on BLG. (a) Overview ADF image of CsI 2D crystals on BLG. Double-layer crystals marked by yellow arrows. (b) Magnified single-layer and (c) double-layer crystal. (d) Plot of the line profile indicated in (c). Green, red, and blue lines indicate the intensity of the substrate and first and second layer. (e) HRTEM image of 2D cesium iodide crystal oriented along $\langle 100\rangle$ on bilayer graphene. (f) FFT corresponding to $(\mathrm{e})$, with marked reflection spots denoting crystallographic information. (g, h) Intensity profiles of the red line $(\{110\}$ plane) and blue line ( $\{100\}$ plane) of the $2 \mathrm{D}$ crystal shown in $(\mathrm{e}) .(\mathrm{i}, \mathrm{j})$ Ball-and-stick model of a single-layer $2 \mathrm{D}$ cesium iodide crystal in [001] and [100] projections (green: Cs, red: I).

Cs and I adatoms are formed due to continuous decomposition of the 3D CsI nanoparticles under the electron irradiation. Later on, under prolonged electron beam irradiation, the residual Cs and I atoms undergo rearrangement and form ordered 2D nanocrystals. One such formation of ordered 2D crystals can be followed in subsequent TEM images (see SI Figure S2.1). Here, the electron beam plays an important role of providing the required energy for the rearrangement on the surface of BLG until the formation of an energetically favorable $2 \mathrm{D}$ crystal takes shape. For instance, in one image stack (see SI-video S2.1), over a period of $62 \mathrm{~s}$, an atomic cluster of 15 atoms transitions several times between an ambiguous disordered structure and an ordered, 2D crystal of cubic symmetry. Unlike the initially formed 3D CsI nanocrystals, the structure of the resulting 2D CsI crystals is robust under electron irradiation and only undergoes rearrangement of atoms rather than rapidly losing material via a radiolysis mechanism.

The $2 \mathrm{D}$ crystals contain between 25 and 180 atoms. Observed by annular dark field (ADF)-STEM, the majority have uniform brightness with the exception of a few that appear brighter (Figure 2a, brighter crystals marked with yellow arrows). Because the intensity in $\mathrm{ADF}$ images scales linearly with the thickness of the specimen, the crystals appearing brighter are thicker. Based on the measured intensities of individual adatoms, we identify them as double-layered, while the less bright crystals are clearly one layer thick (Figure $2 b-d$ ). The intensity profile of the multilayered crystal (Figure 2c,d) clearly shows that these consist of a double layer of CsI.

In Figure $2 \mathrm{~b}$ and e ADF and HRTEM images of single 2D crystals are shown. The FFT of Figure 2e is shown in Figure 2f, where $\{200\}$-CsI reflections at $\sim 0.25 \mathrm{~nm}$ and $\{110\}$ reflections at $\sim 0.35 \mathrm{~nm}$ viewed down the [001] axis are exhibited. In Figure $2 \mathrm{~g}$,h we show the intensity profiles of the red line ( $\{110\}$ plane) and blue line ( $\{100\}$ plane) of the $2 \mathrm{D}$ crystal shown in Figure 2e. Atomic models projected along the [001] and [100] zone axes are shown in Figure 2i,j. This projection along the $\langle 100\rangle$ direction of the atomic structure of the $2 \mathrm{D}$ crystals closely matches bulk cesium iodide, which is of ( $\mathrm{CsCl}-\mathrm{bcc}$ )-type structure. Yet, we observe small deviations in the interplanar spacing along the (100) plane in the 2D CsI nanocrystal ( $a=$ $4.97 \pm 0.23 \AA$ ) in Figure $2 \mathrm{~h}$ compared to the 3D CsI nanocrystal in Figure S1.1b, where the lattice constant is $a=4.54 \pm 0.2 \AA$, which coincides well to the experimentally reported bulk-CsI material. ${ }^{25}$ Further on, we measured interplanar spacing using FFT reflection points from 10 individual 2D CsI nanocrystals from three different samples (a few examples are shown in SI Figure S2.2a), and measurement showed consistent results 
suggesting that the $2 \mathrm{D}$ CsI maintains a nonbulk lattice constant that is larger by $\sim 8 \%$ than its bulk counterpart.

This value depends on the CsI bond length, which is altered in $2 \mathrm{D}$ crystals due to missing neighbor atoms and the interaction with the substrate and also on the arrangement of the atoms in the film vertical to the surface normal. Compared to the bulk value of $0.395 \mathrm{~nm}$, an $11-14 \%$ shorter Cs-I bond length of 0.34 $\mathrm{nm}$ was found for one-dimensional (1D) CsI chains confined inside carbon nanotubes (CNTs), ${ }^{26}$ while a gas phase CsI molecule only has a $0.337 \mathrm{~nm}$ bond distance. ${ }^{27}$ Considering the measured lattice constant of $0.497 \mathrm{~nm}$ in 2D CsI crystals on graphene, and taking into consideration the $b c c$-type CsI crystal structure, we can estimate the Cs-I bond length with epitaxy with BLG to be $\sim 0.43 \mathrm{~nm}$, which is $8 \%$ larger than the bulk CsI crystal (refer to SI Table S1). This deviation of $8 \%$ toward an increase in the Cs-I bond length is opposite the behavior of the CsI bond length in the 1D CsI chain confined in CNTs. In 2D CsI crystals, the interaction with the substrate breaks the symmetry, which leads to a staggered arrangement of the atoms with one species close to graphene and the other further away. The interaction with the graphene thus effectively produces a flattened lattice plane as a result of strain imposed from the preferred binding sites on the substrate that leads to a slightly elongated Cs-I ionic bond distance (refer to Figure S3.2)

To confirm the assigned structure, the chemical identity of the observed atoms needs to be determined. Owing to their similar atomic number, the ${ }^{55} \mathrm{Cs}$ and ${ }^{53} \mathrm{I}$ atoms cannot be distinguished by their contrast in the ADF images (Figure 2b). However, despite the radiation sensitivity, we were able to record atomresolved chemical maps by EELS via the $\mathrm{I}-\mathrm{M}_{4,5}$ and $\mathrm{Cs}-\mathrm{M}_{4,5}$ absorption edges at 620 and $726 \mathrm{eV}$, respectively. An example is shown in Figure 3, panel (a) presenting the survey image of the island that was investigated. Figure $3 \mathrm{~b}$ shows the $\mathrm{ADF}$ image of the same island, which is slightly distorted due to both drift and atom rearrangement during the slow scanning required for the atomically resolved EELS mapping of the radiation-sensitive material. Spectral maps are recorded for I (Figure 3c) and Cs (Figure $3 \mathrm{~d}$ ) by measuring a spectrum at each point and applying multiple linear least-squares fitting (refer to Methods section). The overlay of the map (Figure $3 \mathrm{e}$ ) and even more clearly the processed image (Figure 3f) show the presence of alternating Cs and I atoms. This clearly confirms the proposed ( $\mathrm{CsCl}-\mathrm{bcc})$-type structure. We further find that the outermost atoms of the $2 \mathrm{D}$ crystal are usually $\mathrm{Cs}$ atoms and that the crystal prefers to terminate by atom rows along the $\langle 110\rangle$ direction. Consequently, the number of Cs atoms in the crystal exceeds the number of I atoms. Counting indeed yields different numbers of atoms between the anion and cation sublattice, for instance 44 Cs ions and $37 \mathrm{I}$ ions for the island shown in Figure 4a (refer also to Figure S3).

Assuming fully ionized species, this would cause the $2 \mathrm{D}$ crystals to be net positively charged, which seems energetically unfavorable. Therefore, it suggests that differences in charge transfer with the graphene surface play a key role in the formation of the $2 \mathrm{D}$ crystals. In comparison to SLG, on BLG the interaction between the adsorbed atoms and $\pi$-electrons is enhanced, ${ }^{28}$ resulting in increased binding energy and reduced binding distance of the adsorbed ions to the underlying graphene substrate. As a consequence of this, there will be increased diffusion barriers as the ions feel the corrugation of the surface potential more on BLG than on SLG. The larger density of adatoms and propensity to form stable crystalline agglomerations follows.

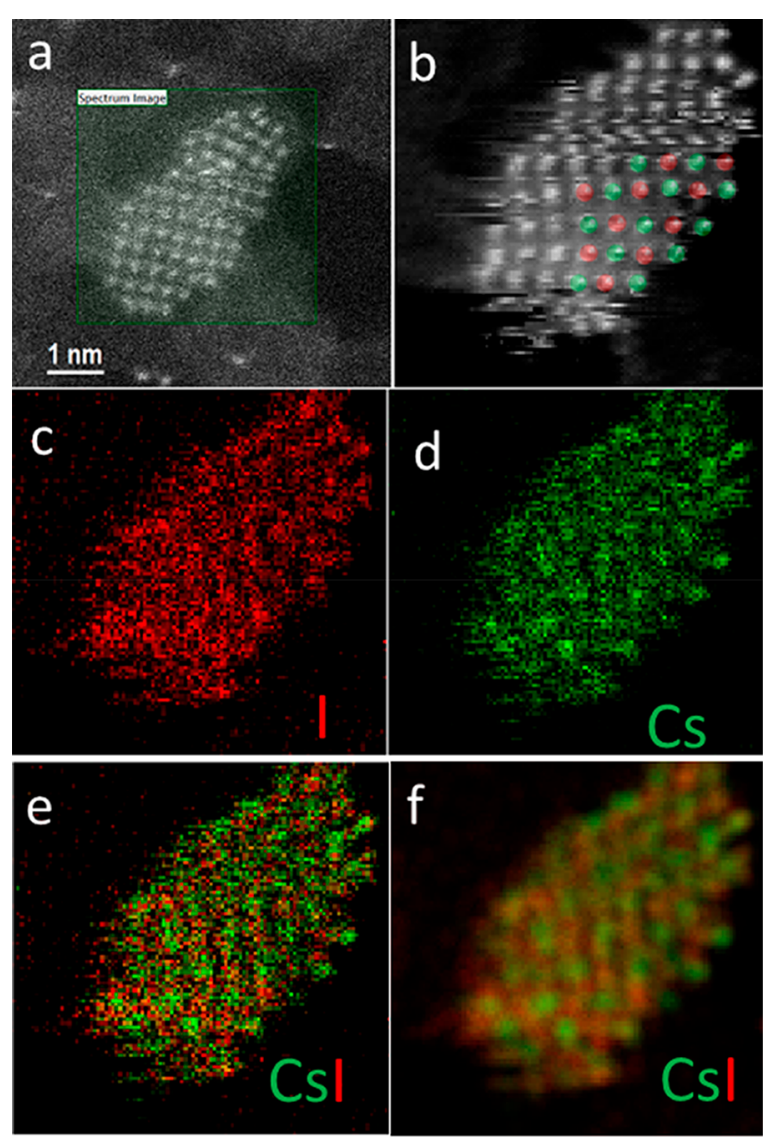

Figure 3. Chemical imaging by EELS mapping with atomic resolution. (a) Survey STEM image. (b) ADF image with chemical identity of Cs and I atoms superimposed; Cs, green, and I, red. (c) I$M_{4,5}$ edge and (d) Cs- $M_{4,5}$ edge elemental map. (e) Superimposed color maps of $I-M_{4,5}\left(\right.$ red) and Cs- $M_{4,5}$ (green) (f) as before with mean filter applied.

Charge transfer between Cs and graphene was calculated to be approximately $0.95 e$ and that between I and graphene to be $0.50 e e^{29,30}$ This suggests a stronger adsorption of Cs to graphene than of I and leads to our assignment of the Cs atoms being closer to the substrate. Also experimental and theoretical evidence in recently reported work on $2 \mathrm{D} \mathrm{NaCl}$ crystals on SLG/BLG ${ }^{31}$ suggests that the presence of additional Na cations stabilizes $2 \mathrm{D}$ crystals via a charge transfer between $\mathrm{Na}$ atoms and aromatic rings of the graphene layers. In the CsI $2 \mathrm{D}$ crystals, additional cations are indeed present, distributed at the edge of the island.

Two-dimensional crystals observed on BLG show dynamic behavior under electron beam exposure. They either rearrange their structure or move as a whole. In the latter case the entire crystal shifts between locations or pivots around one pinning site with little internal rearrangement. These motions occur on time scales where they can be traced in image stacks (refer to SI Video S3.1).

An example of a pinning site is shown in Figure $4 a-d$. A 2D crystal in Figure 4a has disappeared in the subsequent frame (Figure 4d). Only two adsorbed atoms remain visible at the former pivot point (yellow arrows). During electron exposure the $2 \mathrm{D}$ crystal appears to jump between pinning sites (refer to SI Video S3.1). Pinning sites can be lattice defects, in the form of vacancies or dangling bonds, providing the energetically favorable site for a strong interaction with the CsI $2 \mathrm{D}$ lattice. ${ }^{32}$ 

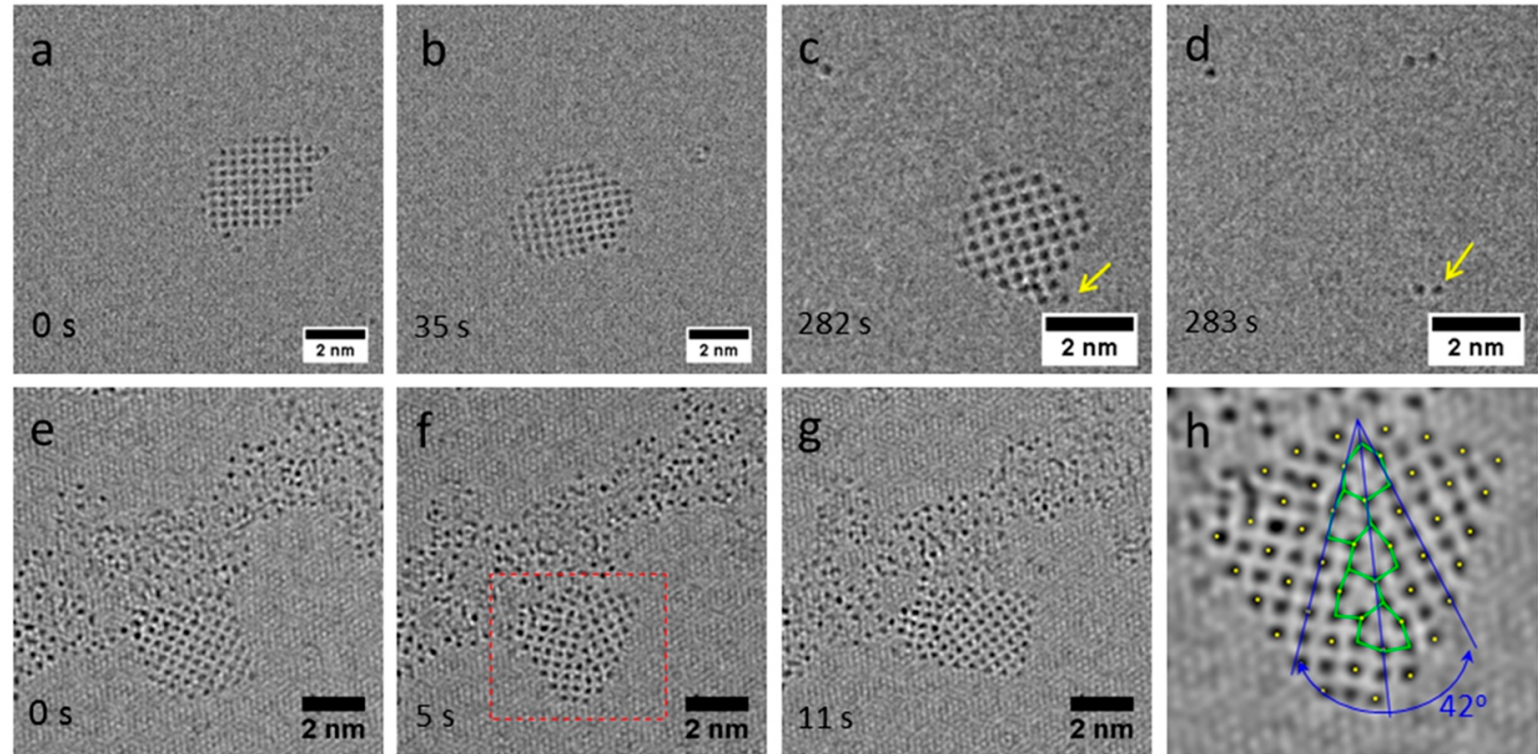

Figure 4. HRTEM images of the dynamics of 2D CsI crystals. $(a-c)$ Rotation and translation of an entire cluster with small rearrangements. (d) After disappearance of the 2D CsI crystal a few atoms remain at the former pinning site (shown by yellow arrow). (e, f, g) Time-sequence transformation of a 2D CsI crystal via a grain boundary under electron beam irradiation. (h) Magnification of the (high-angle) grain boundary in (f). It can be described by a set of distorted hexagonal structural units (green). The grain boundary has a measured tilt angle of 42 degrees.
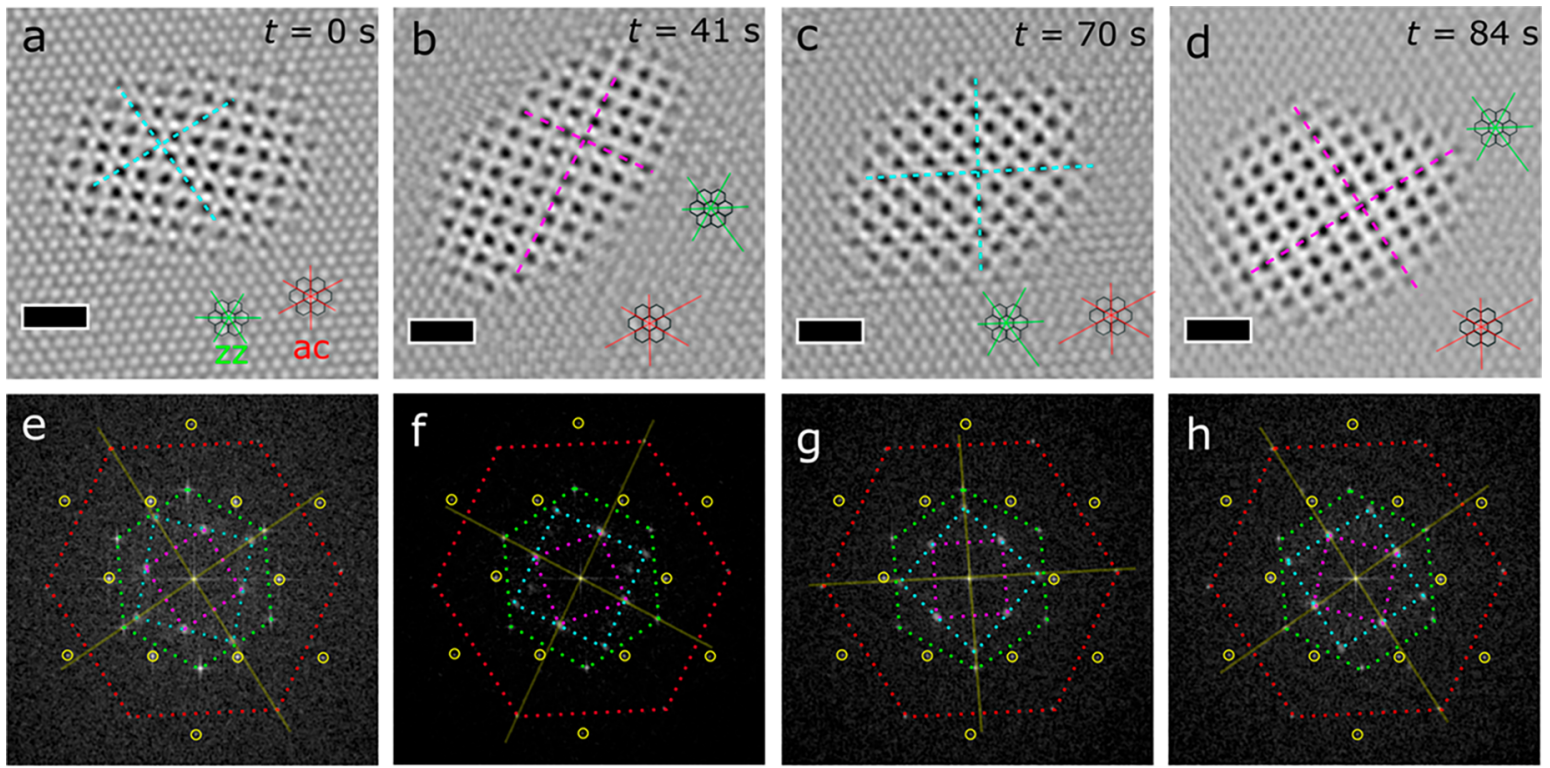

$$
\begin{aligned}
& \text { CsI- }\{200\} \\
& \text { CsI- }\{110\} \ldots \ldots \ldots \\
& 1 L-G r-z z \ldots \ldots . \\
& 1 L-G r-a c \ldots \ldots \ldots \\
& \text { 2L-Gr-spot }
\end{aligned}
$$

Figure 5. Time series of epitaxial orientation of the 2D cesium iodide crystal and underlying graphene sheet. (a-d) Reconstructed HRTEM image (after applying a mask to 2D FFT to S3.1a) of a rotating 2D CsI crystal on graphene (e-h) corresponding FFT patterns showing the detailed relationship between the CsI- $\{200\}$ and $-\{110\}$ planes with respect to the graphene zigzag (Gr-zz) and graphene armchair (Gr-ac) direction. Aligned spots between graphene and the 2D CsI crystal are shown using semitransparent yellow lines. HRTEM images (a-d) reconstructed via Fourier filtering by selecting only the $2 \mathrm{D}$ crystal and one set of graphene lattices (see Supporting Information S3.1). The images (a)-(d) are the slices taken from SI Video S3.1. The scale bar corresponds to $1 \mathrm{~nm}$.

Counting the number of atoms in the $2 \mathrm{D}$ crystal in Figure $4 \mathrm{a}-$ c for different frames reveals a constant number of 81 atoms during the majority of rotations, translations, and rearrangements under the electron beam (see Figure S3). A few frames before its disappearance, the total number of atoms was reduced to $\sim 58$ (Figure 4c).

During rearrangement of $2 \mathrm{D}$ crystals on BLG in the electron beam, grain boundaries often occur. Figure $4 \mathrm{e}-\mathrm{g}$ shows a time 

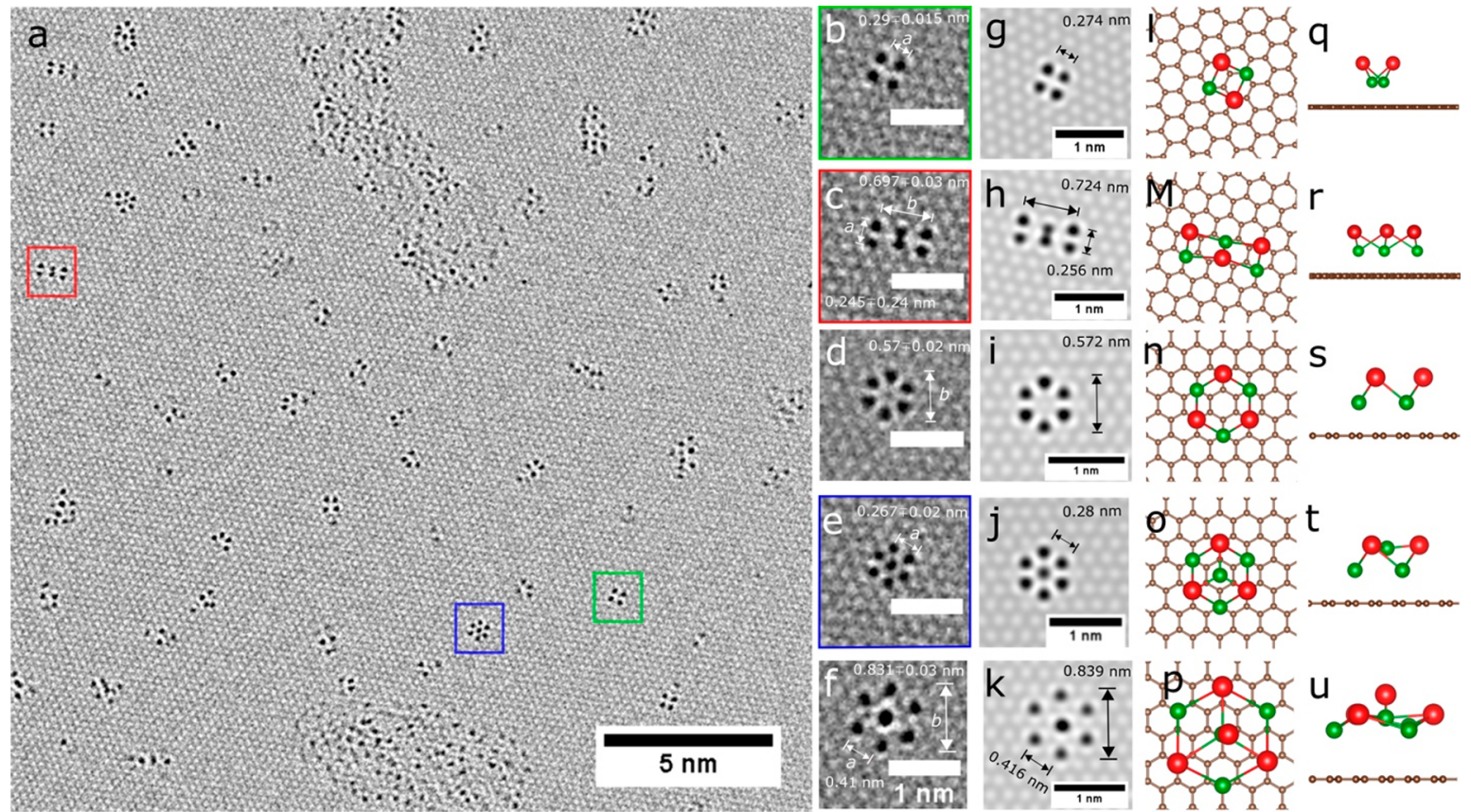

Figure 6. Clusters of CsI on graphene. (a) HRTEM image of SLG with CsI clusters. Four-, 6-, and 7-feature clusters are indicated by green, red, and blue boxes. (b-f) HRTEM images of most commonly occurring 4-, 6-, 7-, and 8-ion clusters. (g-k) Simulated HRTEM image of the corresponding atomic model of the $(4,6,7$, and 8$)$ atom cluster shown in $(1)-(p)$. (q-u) $[100]$ projection of the atomic models shown in $(1)-$ (p). Cesium and iodine atoms are represented by green and red color.

sequence for the formation of a tilt grain boundary (GB) during irradiation at a dose rate of $1.4 \times 10^{6} \mathrm{e}^{-} \mathrm{nm}^{-2} \mathrm{~s}^{-1}$. The GB forms shortly after the exposure starts and becomes distinct after $5 \mathrm{~s}$ to disappear again after $11 \mathrm{~s}$ of observation, leaving behind a rearranged crystal of the same size (refer to SI Video S3.2). A magnified view of the GB is shown in Figure $4 \mathrm{~h}$ of the marked area in Figure $4 \mathrm{f}$ with all Cs ions marked by yellow dots. The core of the grain boundary is composed of structural units resembling distorted hexagons marked in green. The tilt angle measured at the grain boundary is $42^{\circ}$ degrees, which is close to a $\sum 29$ a or a $\sum 73 \mathrm{c}$ tilt GB in bulk crystals. ${ }^{33}$ Equal numbers of Cs and I ions in the grain boundary provide its overall charge neutrality, which contributes to a low-energy structure. ${ }^{34}$ However, here the crystal also terminates with Cs ions at all of its edges.

The intermittent stability of the crystal can be understood in terms of the epitaxial relation to the underlying graphene lattice, as preferred adsorption orientation would lend some stability to the crystal. Figure $5 \mathrm{a}-\mathrm{h}$ shows the epitaxial relationship of the 2D CsI crystal with respect to the underlying graphene sheet. Because we cannot differentiate which of the two graphene layers is in direct contact with the CsI crystal, we selected one of the graphene layers to be compared with the reconstructed HRTEM image of the 2D CsI crystal (Figure 5a-d), noting that a epitaxial relation would be found for either because they have an epitaxial relation themselves (see Supporting Information Figure S3.1). The 2D CsI crystal planes exhibited fluctuating epitaxial rearrangement preferentially aligning with the zigzag or armchair configuration of the graphene lattice, while other orientations were not observed. Owing to the rapid change between these two configurations on the graphene surface, the energy barrier between these two configurations is small. For instance, in Figure 4a and c the (200) (FFT shown in Figure $4 \mathrm{e}, \mathrm{g}$ ) planes of the $2 \mathrm{D}$ CsI crystal are oriented parallel to the graphene zigzag direction and the (020) planes are oriented parallel to the armchair axis. Similarly, in another arrangement in
Figure $4 \mathrm{~b}$ and $\mathrm{d}$ (FFT shown in Figure 4f,h) the (110) planes of the 2D CsI crystal are oriented parallel to the graphene zigzag axis and (101) are oriented parallel to the graphene armchair axis.

CsI Clusters on SLG. Upon electron irradiation of the initial 3D nanocrystals, CsI clusters form in regions of SLG. We observe the Cs and I adatoms arranged in small clusters on the pristine graphene lattice. Larger two-dimensional agglomerations of CsI or even 2D crystals as found on BLG were not observed on SLG. In addition, adatoms decorate amorphous carbon regions randomly. Most clusters consist of 3-10 ions.

A fraction of about $\sim 30 \%$ of the clusters is of regular, recurring structure. We classify regular clusters by the number of 4,6 , or 7 features; examples are highlighted by green, red, and blue squares in Figure 6a. Two types of each 6- and 7-feature clusters are found, shown together with the one 4-feature cluster in magnified TEM micrographs in Figure $6 \mathrm{~b}-\mathrm{f}$.

For the assignment of a structure, we first have to consider the chemical composition of the cluster. The chemical analysis by STEM-EELS fails for these small clusters owing to their limited stability, which leads to rapid movement and decomposition under the focused electron beam. To confirm the chemical composition of the clusters, we repeated the deposition experiment with ion beams of pure $\mathrm{Cs}^{+}$and pure $\mathrm{I}^{-}$ion beams, obtained by mass selection of only the low $m / z$ region. This exclusion of the complementary ion did not yield any clusters on SLG and thus confirms that the observed structures require the presence of both species. We further exclude chemical contamination based on the chemical identification and structural similarity with the $2 \mathrm{D}$ crystals on the BLG regions of the same sample as well as the chemically pure nature of the ES-IBD deposition method. ${ }^{35}$

For an ionic CsI bond, a distance of $0.387 \mathrm{~nm}$ is expected based on measured ionic radii, ${ }^{36} 0.395 \mathrm{~nm}$ is found in bulk crystals, $0.34 \mathrm{~nm}$ in linear CsI in CNTs, and $0.337 \mathrm{~nm}$ for the gas 
phase CsI molecule. As shown in Figure $6 \mathrm{~b}-\mathrm{f}$, the next neighbor distances are measured to range between 0.21 and $0.41 \mathrm{~nm}$, with most distances somewhere around $0.3 \mathrm{~nm}$. This discrepancy indicates that also here we are observing projected bond lengths and that the Cs and I adatoms cannot be arranged in one plane.

Following these initial considerations, we first contemplate the simplest and the most abundant structure observed: the 4feature adsorbate. Due to the discrepancy between CsI bond length and measured distance, we exclude a planar 4-ion cluster as well as a cube shape consisting of 8 ions, and hence we assign a tetrahedral shape. The measured next neighbor distance of 0.29 $\mathrm{nm}$ is close to the Cs-I distance in the (110) planes of the 2D crystals $(0.35 \mathrm{~nm})$ shown in Figure $2 \mathrm{~g}, \mathrm{j}$, which confirms our assumptions about the tetrahedral structure and suggests an even larger vertical distance of the Cs and I planes as compared to the 2D crystal or a shorter Cs-I bond length as in gas phase CsI. The structure consequently has the shape of a tetrahedron standing on one of its four edges, as illustrated in Figure 61,q. Like in the $2 \mathrm{D}$ crystals, the Cs ions are close to the surface. The charge transfer between $\mathrm{Cs}$ and graphene induces a sizable negative partial charge in the $\mathrm{C}$ atoms surrounding the cluster, which stabilizes the position of the I ions at greater distance.

Averaging several HRTEM images reduces the image noise and allows us to relate the position of Cs and I ions to the $\mathrm{C}$ positions of the graphene lattice. Cs and I ions are located at positions close to a $\mathrm{C}$ atom, on a top site or between top and bridge sites (Figure S4). Similar behavior is also found for the larger clusters. Despite these preferred interaction sites, pristine graphene is inert toward adsorbing atoms and molecular species. It is thus not unlikely that the observed CsI atomic clusters on pristine SLG are anchored to defect sites in the graphene lattice that provide an energetically more favorable state. ${ }^{37}$ Clearly the amorphous carbon contamination binds a great number of atoms, which however are individual atoms or irregular clusters. On the clean graphene lattice, a single defect such as a substituted atom or a 5-7 rearrangement would have a much more subtle effect on the potential landscape and TEM image alike. The former would promote the formation of a regular cluster because a small defect would not perturb the cluster structure much. For instance, the four-atom cluster conceals nine carbon atoms in the graphene lattice underneath. The small change in the TEM image, however, will be hard to observe due to the low contrast of the individual $\mathrm{C}$ atom.

For the 6- and 7-feature clusters, we find two different shapes, which are assigned as a rectangular CsI trimer and a ring structure/hexagon with or without central ions (Figure $6 c-f$ ). We found the ring structures to be more stable than the rectangular trimer structure; the latter was found to transform into other structures quickly (refer to SI Video S4.1).

In the TEM image, projected Cs and I positions appear as close as $c=0.245 \pm 0.024 \mathrm{~nm}$ for the central pair of the rectangular cluster, while its other projected Cs-I distances vary greatly with a value of $a=0.29 \pm 0.015 \mathrm{~nm}$ for the outer pairs and $b=0.697 \pm 0.03 \mathrm{~nm}$ for the overall length (hence $0.35 \mathrm{~nm}$ for the separation of the pairs). This large variation, which implies vastly different ion-graphene distances, is found for all other 6- and 7-feature clusters and seems to be related to the interaction with the surface, since in all cases the Cs and I ions seem to be aligned with the positions of the underlying SLG lattice. We find this confirmed by analyzing averaged TEM images. The results are summarized in Figure $61-p$, where we plotted the positions of the cluster ions with respect to the carbon atoms of the SLG.
In all clusters, the heavy Cs and I ions appear as dark contrast over the relatively light contrast of the graphene lattice. With the exception of the central feature of the large 7-feature cluster, the Cs and I atoms have the same size. Slightly distorted shapes of the atoms can be explained by overlaying the contrast of the underlying $\mathrm{C}$ atom, as confirmed by image simulations (Figure $6 \mathrm{~g}-\mathrm{k})$. We rationalize the increased contrast in the large 7feature cluster as Cs-I molecules arranged vertically. In addition to the altered contrast, this shifts the stable positions for the six outer adatoms outward, leading to the large appearance of the cluster.

The observed adsorbed clusters are of unusual size and shape as compared to gas phase clusters. Our initial deposition beam in fact used gas phase clusters of different size, but they merge into 3D crystals upon contact with the surface from which the adsorbed low-dimensional structures are produced by electronbeam irradiation.

Gas phase alkali halide clusters, neutral and charged, can be produced by a variety of methods and are usually detected using mass spectrometry. ${ }^{38}$ The structure of gas phase clusters has been a subject of intense investigation in particular since the discovery of magic numbers, i.e., cluster sizes that are particularly stable due to the special geometry they can adopt. Computational studies were able to assign the mass spectrometric investigations and found which cluster shape is stable for a given number of atoms/ions. ${ }^{39,40}$ For instance, the cube of $27=3^{3}$ ions ( 13 negative and 14 positive) is one of the most stable and hence most prominent cluster ions. The structure of small neutral clusters can also be predicted. For instance, a four-ion cluster of CsI is expected to have square planar structure ${ }^{38,39}$ while a sixion cluster would likely form a planar hexagon, but could also adopt a rectangular trimer shape, which is considered to be unstable. ${ }^{39}$ It has been observed by STM that the presence of a surface can dictate the formation of magic number clusters for molecules, as observed for borazine derivatives on $\mathrm{Au}$ and $\mathrm{Cu}$ substrates. ${ }^{41}$ The charge transfer and screening effect favored the formation of a seven-molecule cluster on $\mathrm{Cu}$, while on $\mathrm{Au}$ large islands would grow due to the absence of the charge transfer. Here we have a similar situation, in which the interaction of the ion with the graphene alters the local adsorption environment, leading to the formation of clusters, which could not be generated on any other surface.

\section{CONCLUSION}

The structures observed on the surface of graphene in this study are the consequence of a complex interplay of our deposition method, interactions with the electron beam, and the intrinsic ionic interactions of $\mathrm{Cs}$ and I with the graphene substrate. Starting from small 3D gas phase cluster ions, we deposit large $3 \mathrm{D}$ nanocrystals and finally generate $2 \mathrm{D}$ islands and clusters in the electron beam. The continuous electron beam irradiation is also causing dynamics observed for bilayer and single-layer adsorbed CsI 2D crystals and clusters. However, the clear cause of the dynamic behavior is the electron beam, and hence we can expect the structures to be stable when we are not observing them.

It is likely that similar structures exist for other material systems as well and that they can be observed on graphene or other 2D materials. Graphene may have a special role though: its electronic system clearly interacts with the adsorbed atoms and significantly influences the structure formation. On SLG, the image charge of the ions stabilized the magic number clusters in unexpected geometries, while the 2D CsI crystal structure was 
exclusively found on BLG, presumably due to the electronic charge interaction between cesium ions and $\pi$-electrons present in the additional graphene sheet. This provides extra binding energy between graphene and adsorbed CsI clusters to the ion at the nucleation site and facilitates the formation of long-range ordered $2 \mathrm{D}$ crystals.

In summary, we have demonstrated graphene-layer-dependent morphology variation of cesium iodide clusters when exposed to electron beam irradiation. This study provides crucial insights into the complex behavior of CsI adatoms deposited on graphene and a compelling motivation to fabricate and characterize other 2D alkali halide species and other materials in this environment. The key to these experiments is the preparation of a PMMA-contamination-free graphene surface before deposition and, in a second step, a highly pure deposition via ES-IBD. In contrast, for many experiments, graphene is cleaned in the microscope by the electron beam. While this provides a graphene surface for inspection, it impedes the unambiguous investigation of the adsorbate behavior, either because deposition is not possible or because the adsorbate would have interacted with contaminations instead of the graphene.

\section{METHODS}

Graphene Transfer on a TEM Grid. PMMA-free graphene suspended on a TEM grid was formed similar to the method described by Longchamp et al. ${ }^{42}$ Commercially available CVD-grown graphene on a copper foil (Graphenea S.A.) was used throughout our experiments. As the first step, a $150 \mathrm{~nm}$ thick PMMA film was spincoated onto the graphene on $\mathrm{Cu}$. The copper film was then etched by floating the PMMA-SLG-Cu assembly in an etching solution ( $8 \mathrm{~g}$ of ammonium persulfate in $100 \mathrm{~mL}$ of deionized water). After $\mathrm{Cu}$ etching, the PMMA-graphene stack was transferred to a deionized water bath for removing the APS solution residue. Subsequently, the stack was transferred onto a platinum-coated (thickness $\sim 15 \mathrm{~nm}$ ) perforated silicon nitride TEM grid. In order to remove the PMMA, the TEM grid was annealed in ambient air at $300^{\circ} \mathrm{C}$ for $30 \mathrm{~min}$, whereupon the thin platinum layer catalyzes the oxidation of the PMMA.

HRTEM, STEM, and EELS. AC-HRTEM was performed using a JEOL ARM200F TEM equipped with a cold-field emission gun and a postspecimen spherical aberration corrector (Cs) operated at an acceleration voltage of $80 \mathrm{kV}$. The spherical aberration was tuned to approximately $1 \mu \mathrm{m}$, and the imaging was done at under-focus conditions, leading to atoms appearing dark. HRTEM image simulations were conducted using the multislice QSTEM software package. ${ }^{43}$ For image simulations, the spherical aberration Cs was set to $1 \mu \mathrm{m}$, the acceleration voltage to $80 \mathrm{kV}$, and the defocus to $-2.5 \mathrm{~nm}$, corresponding to the Scherzer defocus. From the obtained images, several quantities were extracted.

For STEM and EELS, we used a JEOL ARM200F equipped with a DCOR corrector (CEOS GmbH), a cold-field emission electron gun, and a Gatan GIF Quantum ERS electron energy-loss spectrometer. The acceleration voltage was set to $60 \mathrm{kV}$ to minimize the knock-on damage to the graphene. The probe semiconvergence angle of $33.5 \mathrm{mrad}$ was used for both STEM imaging and spectrum imaging. The ADF-STEM images were acquired using medium-angle annular-dark-field imaging conditions, where the measured inner and outer angle of the detector was 42.5 and $92.3 \mathrm{mrad}$, respectively. The EELS inner collection angle was $85 \mathrm{mrad}$. The EELS spectrometer was set to $0.325 \mathrm{eV}$ per channel dispersion. The pixel dwell time for the spectrum imaging was set to $0.01 \mathrm{~s}$. The EELS data were processed with a multivariate weighted principal component analysis routine (MSA Plugin in Digital Micrograph). ${ }^{44}$

Electrospray Ion-Beam Deposition (ES-IBD). The electrospray ion-beam deposition setup is used to generate intact negative gas phase ions of $(\mathrm{CsI})_{n} \mathrm{Cs}^{+}$and deposit them on the graphene substrate under vacuum. The analyte solution $\left(\sim 10-4 \mathrm{~mol} \mathrm{~L}^{-1} \mathrm{CsI}\right.$ in acetonitrile) is pumped through an emitter capillary held at a high electric potential difference of $2-3 \mathrm{kV}$ with respect to the vacuum transfer capillary. A fine mist of charged droplets containing the analyte molecule is emitted. The ions are generated under ambient conditions through an iterative Coulombic fission, while the solvent evaporates. The ions pass through four differential pumping stages to the deposition target held at 10-6 mbar. On their way, radio frequency (rf)-ion optics collimate the beam with an ion funnel in the first and an rf-only quadrupole in the second stage. A further rf-quadrupole is used as a mass filter to generate a chemically pure beam of the desired ion, which is confirmed by an integrated TOF mass spectrometer before the beam is used for deposition. The salient feature of ES-IBD is that the collision energy can be controlled and thereby the interaction of the molecule with the surface. Most importantly, molecules can be soft-landed destructionfree onto the surface or collided at high energies to deliberately cause fragmentation or surface defects. Further, online current monitoring allows for precise knowledge of the deposited charge and thus for the control of the deposition coverage. Further details of the ES-IBID technique are described elsewhere. ${ }^{45}$

The presence of a nearby substrate is an essential influence for the outcome of the cluster and nanocrystal formation. The investigation of cluster and crystal formation at a surface is therefore highly complicated because the adsorption of and growth at an interface is prone to be influenced by contamination. To be able to distinguish the influence of the impurities, the environment must be highly clean during the formation process and in addition compatible with high-resolution imaging methods. ${ }^{19}$

\section{ASSOCIATED CONTENT}

\section{SI Supporting Information}

The Supporting Information is available free of charge at https://pubs.acs.org/doi/10.1021/acsnano.9b10053.

Mass spectrum of deposited CsI clusters, crystal structure of 3D CsI, formation of 2D CsI crystal, difference between SLG and BLG, counting number of Cs and I atoms, fast Fourier filtering of BLG, table comparing CsI bond lengths and mapping position of cluster atoms on graphene (PDF)

Video S1.1 Decomposition of 3D CsI nanoparticles (AVI)

Video S1.2 Loss of crystallinity in 3D CsI nanoparticles (AVI)

Video S2.1 Rearrangement of atoms in 2D CsI crystal (AVI)

Video S3.1 Dynamics of 2D CsI crystal on BLG (AVI)

Video S3.2 Grain boundary formation (AVI)

Video S4.1 Transformation of an atomic cluster (AVI)

\section{AUTHOR INFORMATION}

\section{Corresponding Author}

Nilesh Vats - Max Planck Institute for Solid State Research, D70569 Stuttgart, Germany; 이이이.org/0000-0001-65184603; Email: N.Vats@fkf.mpg.de

\section{Authors}

Yi Wang - Max Planck Institute for Solid State Research, D70569 Stuttgart, Germany

Suman Sen - Max Planck Institute for Solid State Research, D70569 Stuttgart, Germany

Sven Szilagyi - Max Planck Institute for Solid State Research, D70569 Stuttgart, Germany

Hannah Ochner - Max Planck Institute for Solid State Research, D-70569 Stuttgart, Germany 
Sabine Abb - Max Planck Institute for Solid State Research, D70569 Stuttgart, Germany; 이이. orid.org/0000-0002-53604719

Marko Burghard - Max Planck Institute for Solid State Research, D-70569 Stuttgart, Germany

Wilfried Sigle - Max Planck Institute for Solid State Research, D70569 Stuttgart, Germany

Klaus Kern - Max Planck Institute for Solid State Research, D70569 Stuttgart, Germany; Institut de Physique de la Matiere Condensée, Ecole Polytechnique Féderale de Lausanne, CH-1015 Lausanne, Switzerland

Peter A. van Aken - Max Planck Institute for Solid State Research, D-70569 Stuttgart, Germany

Stephan Rauschenbach - Max Planck Institute for Solid State Research, D-70569 Stuttgart, Germany; Department of Chemistry, University of Oxford, Oxford OX1 3TA, United Kingdom

Complete contact information is available at:

https://pubs.acs.org/10.1021/acsnano.9b10053

\section{Author Contributions}

N.V. prepared the graphene samples for deposition and performed HRTEM, multislice image simulations, and data analysis. Y.W. performed high-resolution STEM/EELS experiments and related data processing. S.S. along with S.A., S.S., and H.O. carried out the ESI deposition and mass spectrometry analysis. P.A.v.A., W. S., and S.R. supervised the project. All authors discussed results at all stages, and the final manuscript was written through contributions of all authors. All authors have given approval to the final version of the manuscript.

Notes

The authors declare no competing financial interest.

\section{ACKNOWLEDGMENTS}

We acknowledge the financial support from the European Union's Horizon 2020 research and innovations program under Grant Agreement No. 823717-ESTEEM3.

\section{REFERENCES}

(1) Haider, M. Correction of the Spherical Aberration of a $200 \mathrm{kV}$ TEM by Means of a Hexapole Corrector. Optik 1995, 99, 167-179.

(2) Batson, P. E.; Dellby, N.; Krivanek, O. L. Sub-Ångstrom Resolution Using Aberration Corrected Electron Optics. Nature 2002, 418, 617.

(3) Kaiser, U.; Biskupek, J.; Meyer, J. C.; Leschner, J.; Lechner, L.; Rose, H.; Stöger-Pollach, M.; Khlobystov, A. N.; Hartel, P.; Müller, H.; Haider, M.; Eyhusen, S.; Benner, G. Transmission Electron Microscopy at $20 \mathrm{kV}$ for Imaging and Spectroscopy. Ultramicroscopy 2011, 111, $1239-1246$.

(4) Schäffel, F.; Wilson, M.; Warner, J. H. Motion of Light Adatoms and Molecules on the Surface of Few-Layer Graphene. ACS Nano 2011, 5, 9428-9441.

(5) Ke, X.; Turner, S.; Quintana, M.; Hadad, C.; Montellano-López, A.; Carraro, M.; Sartorel, A.; Bonchio, M.; Prato, M.; Bittencourt, C.; Van Tendeloo, G. Dynamic Motion of Ru-Polyoxometalate Ions (POMs) on Functionalized Few-Layer Graphene. Small 2013, 9, 3922-3927.

(6) Dubey, G.; Urcuyo, R.; Abb, S.; Rinke, G.; Burghard, M.; Rauschenbach, S.; Kern, K. Chemical Modification of Graphene via Hyperthermal Molecular Reaction. J. Am. Chem. Soc. 2014, 136, 13482-13485.

(7) Markevich, A.; Kurasch, S.; Lehtinen, O.; Reimer, O.; Feng, X.; Müllen, K.; Turchanin, A.; Khlobystov, A. N.; Kaiser, U.; Besley, E.
Electron Beam Controlled Covalent Attachment of Small Organic Molecules to Graphene. Nanoscale 2016, 8, 2711-2719.

(8) Chamberlain, T. W.; Biskupek, J.; Skowron, S. T.; Markevich, A. V.; Kurasch, S.; Reimer, O.; Walker, K. E.; Rance, G. A.; Feng, X.; Müllen, K.; Turchanin, A.; Lebedeva, M. A.; Majouga, A. G.; Nenajdenko, V. G.; Kaiser, U.; Besley, E.; Khlobystov, A. N. StopFrame Filming and Discovery of Reactions at the Single-Molecule Level by Transmission Electron Microscopy. ACS Nano 2017, 11, 25092520.

(9) Skowron, S. T.; Chamberlain, T. W.; Biskupek, J.; Kaiser, U.; Besley, E.; Khlobystov, A. N. Chemical Reactions of Molecules Promoted and Simultaneously Imaged by the Electron Beam in Transmission Electron Microscopy. Acc. Chem. Res. 2017, 50, 17971807.

(10) Meyer, J. C.; Eder, F.; Kurasch, S.; Skakalova, V.; Kotakoski, J.; Park, H. J.; Roth, S.; Chuvilin, A.; Eyhusen, S.; Benner, G.; Krasheninnikov, A. V.; Kaiser, U. Accurate Measurement of Electron Beam Induced Displacement Cross Sections for Single-Layer Graphene. Phys. Rev. Lett. 2012, 108, 196102.

(11) Ramasse, Q. M.; Zan, R.; Bangert, U.; Boukhvalov, D. W.; Son, Y.-W.; Novoselov, K. S. Direct Experimental Evidence of MetalMediated Etching of Suspended Graphene. ACS Nano 2012, 6, 40634071.

(12) Wang, W. L.; Santos, E. J. G.; Jiang, B.; Cubuk, E. D.; Ophus, C.; Centeno, A.; Pesquera, A.; Zurutuza, A.; Ciston, J.; Westervelt, R.; Kaxiras, E. Direct Observation of a Long-Lived Single-Atom Catalyst Chiseling Atomic Structures in Graphene. Nano Lett. 2014, 14, 450455.

(13) Chen, Q.; He, K.; Robertson, A. W.; Kirkland, A. I.; Warner, J. H. Atomic Structure and Dynamics of Epitaxial 2D Crystalline Gold on Graphene at Elevated Temperatures. ACS Nano 2016, 10, 1041810427.

(14) Wang, S.; Li, H.; Zhang, J.; Guo, S.; Xu, W.; Grossman, J. C.; Warner, J. H. Epitaxial Templating of Two-Dimensional Metal Chloride Nanocrystals on Monolayer Molybdenum Disulfide. ACS Nano 2017, 11, 6404-6415.

(15) Robertson, A. W.; Lee, G.-D.; Lee, S.; Buntin, P.; Drexler, M.; Abdelhafiz, A. A.; Yoon, E.; Warner, J. H.; Alamgir, F. M. Atomic Structure and Dynamics of Epitaxial Platinum Bilayers on Graphene. ACS Nano 2019, 13 (10), 12162-12170.

(16) Song, C.-L.; Sun, B.; Wang, Y.-L.; Jiang, Y.-P.; Wang, L.; He, K.; Chen, X.; Zhang, P.; Ma, X.-C.; Xue, Q.-K. Charge-Transfer-Induced Cesium Superlattices on Graphene. Phys. Rev. Lett. 2012, 108, 156803.

(17) Fenn, J. B.; Mann, M.; Meng, C. K.; Wong, S. F.; Whitehouse, C. M. Electrospray Ionization for Mass Spectrometry of Large Biomolecules. Science 1989, 246, 64-71.

(18) Ouyang, Z.; Takáts, Z.; Blake, T. A.; Gologan, B.; Guymon, A. J.; Wiseman, J. M.; Oliver, J. C.; Davisson, V. J.; Cooks, R. G. Preparing Protein Microarrays by Soft-Landing of Mass-Selected Ions. Science 2003, 301, 1351-1354.

(19) Vats, N.; Rauschenbach, S.; Sigle, W.; Sen, S.; Abb, S.; Portz, A.; Dürr, M.; Burghard, M.; van Aken, P. A.; Kern, K. Electron Microscopy of Polyoxometalate Ions on Graphene by Electrospray Ion Beam Deposition. Nanoscale 2018, 10, 4952-4961.

(20) Gerlach, J. W.; Schumacher, P.; Mensing, M.; Rauschenbach, S.; Cermak, I.; Rauschenbach, B. Ion Mass and Energy Selective Hyperthermal Ion-Beam Assisted Deposition Setup. Rev. Sci. Instrum. 2017, 88, 063306.

(21) Egerton, R. F.; Li, P.; Malac, M. Radiation Damage in the TEM and SEM. Micron 2004, 35, 399-409.

(22) Hobbs, L. W. Transmission Electron Microscopy of Extended Defects in Alkali Halide Crystals. Surf. Defect Prop. Solids. 1975, 4, 152 250.

(23) Khlobystov, A. N. Carbon Nanotubes: From Nano Test Tube to Nano-Reactor. ACS Nano 2011, 5, 9306-9312.

(24) Geng, X.; Sun, W.; Wu, W.; Chen, B.; Al-Hilo, A.; Benamara, M.; Zhu, H.; Watanabe, F.; Cui, J.; Chen, T.-p. Pure and Stable Metallic Phase Molybdenum Disulfide Nanosheets for Hydrogen Evolution Reaction. Nat. Commun. 2016, 7, 10672. 
(25) Smakula, A.; Kalnajs, J. Precision Determination of Lattice Constants with a Geiger-Counter X-Ray Diffractometer. Phys. Rev. 1955, 99, 1737-1743.

(26) Senga, R.; Komsa, H.-P.; Liu, Z.; Hirose-Takai, K.; Krasheninnikov, A. V.; Suenaga, K. Atomic Structure and Dynamic Behaviour of Truly One-Dimensional Ionic Chains inside Carbon Nanotubes. Nat. Mater. 2014, 13, 1050.

(27) Viswanathan, R.; Hilpert, K. Mass Spectrometric Study of the Vaporization of Cesium Iodide and Thermochemistry of (CsI)2(g) and (CsI)3(g). Berichte der Bunsengesellschaft für physikalische Chemie 1984, $88,125-131$.

(28) Hardcastle, T. P.; Seabourne, C. R.; Zan, R.; Brydson, R. M. D.; Bangert, U.; Ramasse, Q. M.; Novoselov, K. S.; Scott, A. J. Mobile Metal Adatoms on Single Layer, Bilayer, and Trilayer Graphene: An Ab Initio DFT Study with van der Waals Corrections Correlated with Electron Microscopy Data. Phys. Rev. B: Condens. Matter Mater. Phys. 2013, 87, 195430.

(29) Davydov, S. Y.; Sabirova, G. I. Adsorption of Hydrogen, Alkali Metal, and Halogen Atoms on Graphene: Adatom Charge Calculation. Tech. Phys. Lett. 2011, 37, 515-518.

(30) Rytkönen, K.; Akola, J.; Manninen, M. Density Functional Study of Alkali-Metal Atoms and Monolayers on Graphite (0001). Phys. Rev. B: Condens. Matter Mater. Phys. 2007, 75, 075401.

(31) Shi, G.; Chen, L.; Yang, Y.; Li, D.; Qian, Z.; Liang, S.; Yan, L.; Li, L. H.; Wu, M.; Fang, H. Two-Dimensional Na-Cl Crystals of Unconventional Stoichiometries on Graphene Surface from Dilute Solution at Ambient Conditions. Nat. Chem. 2018, 10, 776-779.

(32) Banhart, F.; Kotakoski, J.; Krasheninnikov, A. V. Structural Defects in Graphene. ACS Nano 2011, 5, 26-41.

(33) Mykura, H.; Bansal, P. S.; Lewis, M. H. Coincidence-Site-Lattice Relations for MgO-CdO Interfaces. Philos. Mag. A 1980, 42, 225-233.

(34) Duffy, D. M. Grain Boundaries in Ionic Crystals. J. Phys. C: Solid State Phys. 1986, 19, 4393.

(35) Portz, A.; Baur, M.; Rinke, G.; Abb, S.; Rauschenbach, S.; Kern, K.; Dürr, M. Chemical Analysis of Complex Surface-Adsorbed Molecules and Their Reactions by Means of Cluster-Induced Desorption/Ionization Mass Spectrometry. Anal. Chem. 2018, 90, $3328-3334$

(36) Shannon, R. Revised Effective Ionic Radii and Systematic Studies of Interatomic Distances in Halides and Chalcogenides. Acta Crystallogr., Sect. A: Cryst. Phys., Diffr., Theor. Gen. Crystallogr. 1976, 32, $751-767$.

(37) Robertson, A. W.; Montanari, B.; He, K.; Kim, J.; Allen, C. S.; Wu, Y. A.; Olivier, J.; Neethling, J.; Harrison, N.; Kirkland, A. I.; Warner, J. H. Dynamics of Single Fe Atoms in Graphene Vacancies. Nano Lett. 2013, 13, 1468-1475.

(38) Martin, T. P. Alkali Halide Clusters and Microcrystals. Phys. Rep. 1983, 95, 167-199.

(39) Diefenbach, J.; Martin, T. P. Model Calculations for Alkali Halide Clusters. J. Chem. Phys. 1985, 83, 4585-4590.

(40) Fernandez-Lima, F. A.; Becker, C.; Gillig, K.; Russell, W. K.; Nascimento, M. A. C.; Russell, D. H. Experimental and Theoretical Studies of (CsI)nCs+ Cluster Ions Produced by $355 \mathrm{~nm}$ Laser Desorption Ionization. J. Phys. Chem. A 2008, 112 (44), 11061-11066.

(41) Kervyn, S.; Kalashnyk, N.; Riello, M.; Moreton, B.; Tasseroul, J.; Wouters, J.; Jones, T. S.; De Vita, A.; Costantini, G.; Bonifazi, D. Magic" Surface Clustering of Borazines Driven by Repulsive Intermolecular Forces. Angew. Chem., Int. Ed. 2013, 52, 7410-7414.

(42) Longchamp, J.-N.; Escher, C.; Fink, H.-W. Ultraclean Freestanding Graphene by Platinum-Metal Catalysis. J. Vac. Sci. Technol., B: Nanotechnol. Microelectron.: Mater., Process., Meas., Phenom. 2013, 31, 020605.

(43) Koch, C. T. Determination of Core Structure Periodicity and Point Defect Density along Dislocations. Ph.D. Thesis, Arizona State University, AZ, 2002.

(44) Bosman, M.; Watanabe, M.; Alexander, D. T. L.; Keast, V. J. Mapping Chemical and Bonding Information Using Multivariate Analysis of Electron Energy-Loss Spectrum Images. Ultramicroscopy 2006, 106, 1024-1032.
(45) Rauschenbach, S.; Stadler, F. L.; Lunedei, E.; Malinowski, N.; Koltsov, S.; Costantini, G.; Kern, K. Electrospray Ion Beam Deposition of Clusters and Biomolecules. Small 2006, 2, 540-547. 